\title{
Balancing Fidelity and Flexibility: Usual Care for Young Children With an Increased Likelihood of Having Autism Spectrum Disorder Within an Early Intervention System
}

\author{
Katherine Pickard ${ }^{1,3}$ (D) Hannah Mellman ${ }^{1} \cdot$ Kyle Frost $^{2} \cdot$ Judy Reaven ${ }^{1} \cdot$ Brooke Ingersoll $^{2}$ \\ Accepted: 9 January 2021 / Published online: 23 January 2021 \\ (c) The Author(s), under exclusive licence to Springer Science+Business Media, LLC part of Springer Nature 2021
}

\begin{abstract}
Naturalistic developmental behavioral interventions (NDBIs) are evidence-based interventions for young children with autism spectrum disorder. There has been growing interest in implementing manualized NDBIs within the early intervention (EI) system without a clear understanding of how these programs and the broader strategies encompassed within them are already used by EI providers. This study examined the use of manualized NDBI programs and broader NDBI strategies within an EI system and factors that impacted their use. Eighty-eight EI providers completed a measure of NDBI program and strategy use. Thirty-three providers participated in a supplemental focus group or interview. Overall, providers described using broader NDBI strategies and the need to adapt manualized NDBI programs. Provider-, intervention-, and organization-level factors impacted their use of NDBI programs and strategies.
\end{abstract}

Keywords Autism spectrum disorder · Naturalistic developmental behavioral interventions · Early intervention · Adaptation · Implementation

\section{Introduction}

The prevalence of autism spectrum disorder (ASD) has continued to increase with current estimates that 1 in 54 children are born with the neurodevelopmental disability (Maenner et al. 2020). The rise in ASD prevalence has been paralleled by advances in the early identification of ASD (Pierce et al. 2019), thereby opening the door to earlier opportunities for intervention during a window of optimal brain development and plasticity (Dawson 2008; Fox et al. 2010). Parent-mediated interventions that use a blend of Naturalistic, Developmental and Behavioral Intervention strategies (NDBIs) are one example of an evidence-based early intervention

Katherine Pickard

katherine.e.pickard@emory.edu

1 JFK Partners, University of Colorado Anschutz Medical Campus, Aurora, CO, USA

2 Department of Psychology, Michigan State University, East Lansing, Michiga, USA

3 Division of Autism and Related Disabilities, Department of Pediatrics, Emory School of Medicine, 1920 Briarcliff Road, Atlanta, GA 30329, USA model for young children with ASD. NDBIs are designed to capitalize on the powerful role of caregivers in social communication development by building reciprocal interactions between caregivers and their children with the goal to foster social engagement and naturalistic teaching opportunities (Schreibman et al. 2015).

Although the outcomes associated with NDBIs are promising (Nevill et al. 2018; Trembath et al. 2019), accessing these intervention models within community settings is often contingent on having a medical diagnosis of ASD. Unfortunately, this contingency is at odds with the capacity to identify ASD symptoms and to diagnose ASD. Currently, on average, children receive an ASD diagnosis for the first time at 51 months, and fewer than half of children obtain this diagnosis prior to 36 months (Maenner et al. 2020). A number of factors, including long wait lists to obtain medical diagnoses, parent- and provider-perceptions of child development, and structural and systemic barriers may contribute to delays in accessing an early ASD diagnosis and, subsequently, early intervention services (Angell et al. 2018; Sanchez-Garcia et al. 2019; Sheldrick et al. 2019).

While there are a number of efforts underway to address gaps in the early identification of ASD and ASD risk (Eisenhower et al. 2020; Pierce et al. 2019), providing access to 
early interventions for ASD and positive outcomes for young children and their families is essential. To do so, it is imperative to deliver evidence-based early interventions within systems of care that support both young children with ASD in addition to those who have an increased chance of having ASD (Bottema-Beutel et al. 2020), but who may not have a medical diagnosis (Vivanti et al. 2018). The early intervention (EI) system is one example of a system that is well positioned to do this, as it is federally mandated to provide services to children birth to three with developmental delays under Part $\mathrm{C}$ of the individuals with disabilities education act (IDEA; Adams and Tapia 2013). In fact, in a recent study that used a multi-tiered screening approach, about ten percent of young children served within the EI system either already had or went on to obtain an ASD diagnosis (Eisenhower et al. 2020). This makes the EI system a first line of intervention for many young children with an increased likelihood of having ASD.

With this in mind, there has been growing interest to embed early ASD interventions within the EI system (Stahmer et al. 2017; Vivanti et al. 2018). Recent research has suggested that, when NDBIs are moved into the EI system, they are perceived as feasible and acceptable by EI providers and may have positive outcomes for families of young children with ASD and social communication delays, even if delivered at somewhat low intensity (Stahmer et al. 2017; Rogers et al. 2020a). This research has been foundational in building an understanding of how to translate NDBIs into systems naturally positioned to serve both children with a known diagnosis of ASD in addition to children with an increased likelihood of having ASD (Vivanti et al. 2018).

In order to continue to translate NDBI programs into the EI system in a viable and sustainable manner, it is also important to understand the intervention practices that young children with ASD and social communication delays already receive within this system. To do so, it may be important to examine the use of manualized NDBI programs, in addition to the broader use of the NDBI strategies encompassed within these programs (Frost et al. 2020; Vibert et al. 2020), as there may be benefits and challenges to using both approaches. On the one hand, manualized interventions may support EI providers in delivering interventions to fidelity, likely fostering stronger outcomes for children and families (Kendall and Beidas 2007). For NDBI programs, a manualized approach may be particularly important when considering that parent learning is at the foundation of the intervention. Manualized interventions often include strategies to support adult learning, including parent-driven goals, coaching and feedback, and sequenced and systematic instruction (e.g., Kaiser and Hancock 2003).

Despite these benefits, it is also quite possible that, in the absence of formal training in manualized NDBI programs, EI providers use broader NDBI strategies that were learned pre-service (Stronach and Schmedding-Bartley 2019). Understanding the degree to which providers use broader NDBI strategies and the relative advantage of doing so is imperative. There is also growing awareness of the variability in resources that are required to learn manualized NDBI programs, with some programs being relatively costly and time intensive to be trained in (Trembath et al. 2019). These factors may impact the fit of these programs within community contexts and may ultimately lead EI providers to circumvent training efforts or to adapt manualized NDBI programs to be delivered in context-consistent ways (Chambers et al. 2013; Stirman et al. 2013).

In fact, given the known tendency towards intervention adaptation (Lau et al. 2017), the ability to flexibly deliver NDBI strategies within the EI system may be ideal. One recent study examined the practicality of using broader NDBI strategies for toddlers with and without ASD within early childhood and daycare settings (Maye et al. 2020). Results demonstrated that providers perceived most NDBI strategies as being effective and practical for both toddlers with and without ASD (Maye et al. 2020). Although this research did not examine providers' use of broader NDBI strategies, it did demonstrate the possible application of these broader strategies outside of children with a known diagnosis of ASD (Maye et al. 2020).

To date, research has yet to examine the existing use of both manualized NDBI programs and broader NDBI strategies by EI providers within community systems. This is a gap that limits our understanding of "usual care" for children being served within the EI system and the more naturalistic capacity for the EI system to serve these children. In addition to expanding our understanding of NDBI program and strategy use within the EI system, it is also critical to understand factors that may impact the translation of manualized NDBI programs and/or strategies into this system. Factors that may be important to consider include those specific to NDBI programs (e.g., compatibility within the EI system, ease of use, relative advantage; e.g., Rogers 2002), factors specific to EI providers (e.g., years of EI experience, self-efficacy and confidence, attitudes towards EBPs; Stewart et al. 2019), and system-level factors (e.g., leadership support, organizational incentive, culture; Damschroder et al. 2009; Glasgow et al. 2019). Without considering these factors, there is an increased risk that translation efforts will fail in part due to providers not adopting or sustaining NDBI programs and/or strategies over time (Vivanti et al. 2018).

With this in mind, the goals of the current study were to examine how young children with ASD and social communication delays are currently served within the EI system, and how best to translate NDBI programs and/or strategies within this system. Specifically, this study used a mixedmethods approach to examine: (1) provider-reported use of both manualized NDBI programs and broader NDBI 
strategies; (2) factors that impact the use of NDBI programs and/or strategies within the EI system; and (3) methods to increase the delivery of NDBI programs and/or strategies within this system of care.

\section{Method}

\section{Participants}

Eighty-eight interdisciplinary Early Intervention providers in Colorado participated in this study. Providers were recruited if they had directly served a child under 36-months of age who either had a medical diagnosis of ASD or whom they suspected had an increased likelihood of having ASD. Participants were excluded if they did not work within Colorado. All participating providers completed an online survey that asked about their use of manualized NDBI programs in addition to broader NDBI strategies. Participants also had the option of participating in a supplemental focus group or individual interview to obtain additional information regarding their use of NDBI programs and strategies. Focus groups also expanded upon the NDBI survey by inquiring about factors that impacted providers' use of NDBI strategies. A total of 33 of the 88 participating providers opted to participate in a supplemental focus group or interview. Participant demographic information is presented in Table 1.

\section{Procedure}

This study was conducted in collaboration with providers and administrators within Colorado's Early Intervention (EI Colorado) program and was approved by the Colorado Multiple Institutional Review Board (i.e., COMIRB). Once the study protocol was approved by COMIRB, information about the study aims and procedures was distributed through email listservs that include Early Intervention providers within Colorado. Interested providers were able to enroll in the research study by emailing the primary investigator, who was able to answer questions and to coordinate the distribution of the online survey and the organization of focus groups and interviews.

\section{Measures}

\section{Demographic Information}

All participants provided basic demographic information including their gender, age, race, ethnicity, educational attainment, discipline (i.e., speech language pathology, occupational therapy, early childhood educator, etc.), and
Table 1 Provider demographic information $(N=88)$

\begin{tabular}{|c|c|c|c|}
\hline & $M(S D)$ & Range & Percent \\
\hline Gender (female) & & & 96.60 \\
\hline Years of EI experience & $11.29(8.64)$ & $1-34$ & \\
\hline \multicolumn{4}{|l|}{ Ethnicity } \\
\hline Hispanic/Latinx & & & 18.2 \\
\hline Non-Hispanic/Latinx & & & 81.8 \\
\hline \multicolumn{4}{|l|}{ Race } \\
\hline Black/African American & & & - \\
\hline White/Caucasian & & & 91.2 \\
\hline Asian & & & 3.4 \\
\hline American Indian & & & 1.1 \\
\hline Native Hawaiian & & & 1.1 \\
\hline Biracial/multiracial & & & 3.2 \\
\hline \multicolumn{4}{|l|}{ Education } \\
\hline Bachelors & & & 18.1 \\
\hline Some graduate/MS & & & 70.5 \\
\hline Professional/PhD & & & 11.4 \\
\hline \multicolumn{4}{|l|}{ Profession } \\
\hline Applied behavior analysis (ABA) & & & 11.4 \\
\hline Speech language pathologist & & & 38.6 \\
\hline Occupational therapist & & & 17.0 \\
\hline Physical therapist & & & 7.8 \\
\hline Psychologist & & & 9.1 \\
\hline Early childhood educator & & & 16.1 \\
\hline
\end{tabular}

years of experience working within the Early Intervention system. Demographic information is displayed in Table 1.

\section{Provider Confidence}

All participants rated their confidence in providing intervention to a young child with ASD or whom they suspected might have ASD. Providers rated this single item (i.e., "how confident do you feel providing services to young children with ASD or whom you suspect may have ASD?") using a five-point Likert scale with a one indicating having no confidence at all and a five indicating feeling very confident.

\section{Early Intervention Program Competence}

Participants rated their perceived competence with a set of 17 manualized early intervention programs for ASD. These programs included manualized NDBIs in addition to other early intervention models that use primarily developmental (e.g., Floortime) or behavioral (e.g., Discrete Trial Training) teaching methods. Participants rated their competence in each program using a five-point Likert scale with a one indicating having no competence in the program and a five indicating feeling very competent in delivering the program. 


\section{NDBI Strategy Survey}

A comprehensive set of defined NDBI intervention elements were developed using an adapted Delphi procedure as part of a previous study (Frost et al. 2020). A first draft of items was developed from the content of several NDBI treatment fidelity forms and available treatment manuals. An expert panel provided open-ended review and commentary, and the items were revised and returned to the expert panel for approval and additional minor revisions. This process was used to design an observational tool that was also adapted to be used as a self-report measure (Frost and Ingersoll 2020). For the current study, providers were presented with the text description of 19 intervention element and asked to report on the percent of their most recent therapy session that they used that technique. Providers were specifically instructed to report on actual use, rather than what they might consider ideal use. An average percentage was calculated for a developmental and behavioral sub-scale. These subscales were created consistent with item loadings in previous research (Frost and Ingersoll 2020). In addition, providers were asked to indicate the best description of their utilization of the strategy: integrated and used throughout the session; interspersed between other techniques; used during "breaks," to help the child warm up, or for positive reinforcement; and as needed.

\section{Focus Groups and Interviews}

Focus groups were semi-structured and included EI providers within the same county or region (i.e., Community Centered Board) in Colorado. Individual interviews were conducted with EI providers when they were not available to participate in a focus group. All participants completed the NDBI Strategy Survey either in-person or online prior to their participation in the focus group or individual interview. The survey was completed beforehand in order to ground participants in the manualized NDBI programs and strategies that were the focus of the interview. Focus groups and interviews were conducted in an iterative process, with qualitative themes from earlier focus groups helping to inform slight modifications to questions asked in later groups. Focus groups and interviews were conducted until saturation was reached. Saturation was defined as the point at which no new themes emerged from either the focus groups or the individual interviews (Guest et al. 2006).

Twenty-eight EI providers and administrators participated in a total of four semi-structured focus groups that lasted between 49 and $72 \min (M=61.67 \mathrm{~min})$. Five providers completed an individual interview that lasted between 25 and $56 \mathrm{~min}(M=34.98 \mathrm{~min})$. All focus groups and interviews were conducted by one or both of the primary authors (KP and HM), each with a graduate degree and experience working with young children with ASD within the EI system. Focus groups and individual interviews were conducted either in-person at the Community-Centered Board or via Zoom in order to maintain the safety of research participants and staff due to the COVID-19 pandemic. Each focus group and interview opened with introductions and an overview of the research project, and solicited feedback about the NDBI survey. Providers were then asked questions tied to: (1) the identification of children at-risk for ASD; (2) the strategies used to support these children; (3) their familiarity with manualized NDBI programs and broader NDBI strategies; (4) the relative utility of learning a manualized NDBI program versus broader NDBI strategies; and (5) factors impacting their use of NDBI programs and/or strategies. Consistent with the qualitative analytic approach, at the end of each interview, two of the primary authors (KP and HM) debriefed to determine whether additional modifications needed to be made to the interview protocol based on the existing data (Hsieh and Shannon 2005; Miles et al. 2014).

\section{Data Analysis}

\section{Quantitative Analyses}

SPSS was used to examine descriptive statistics regarding provider demographic information, perceived NDBI program competency, and descriptions of NDBI strategy use. Pearson's correlation analyses were used to examine the associations between the reported use of developmental and behavioral intervention strategies.

\section{Qualitative Analysis}

All focus groups and individual interviews were de-identified, transcribed verbatim, and checked for accuracy. Transcripts were then analyzed using an iterative process grounded in conventional content analysis (Hsieh and Shannon 2005). In the first step of this process, two of the primary authors reviewed the transcripts to independently generate a list of descriptive codes. These initial codes described data chunks based on the direct and concrete meaning of the text (Hsieh and Shannon 2005; Miles et al. 2014). After independently generating initial codes, the two primary authors came together to come to consensus on code names and definitions, and to finalize a code book. The process of developing the codebook was done iteratively and was guided by constant comparative methodology, in which qualitative data was continuously compared to emerging themes in order to ensure the relevance of the codebook and to make adaptations to code definitions as needed (Creswell 2013). Consensus coding was used for reliability throughout the coding process with discrepancies reviewed by a senior author. 
After applying the code book to all focus groups and interviews, patterns and groupings were identified amongst first-cycle codes (Miles et al. 2014). These groupings were facilitated by the use of MAXQDA software and are described in Table 3. All groupings were compared with the focus group and interview transcripts. Triangulation occurred by comparing qualitative themes to those present within the quantitative survey results.

\section{Results}

\section{Description of NDBI Program and Strategy Use}

Participating providers reported feeling relatively confident providing services for young children with or at-risk for $\operatorname{ASD}(M=4.07 ; S D=0.84)$. On average, providers reported feeling relatively competent delivering at least one early intervention program, irrespective of whether it was an NDBI, with their highest competency rating being $M=3.78$, $S D=1.18$. When looking at NDBIs more specifically, competency ratings were also relatively high for their highest rated NDBI $(M=3.72 S D=1.22)$. Importantly, providers' perceived competency in NDBI programs was significantly and positively associated with their perceived competence in supporting young children with ASD or other social communication delays $(r=0.58 ; p<0.001)$. Mean competency ratings across early intervention programs, including NDBIs, are displayed in Table 2 and ranged from $M=1.23$ $(S D=0.58)$ for Project ImPACT to $M=3.54(S D=1.32)$ for JASPER.

Providers reported the extent to which they deliver broader NDBI strategies to a specific young child with or at-risk for ASD on their caseload. The following NDBI strategies were reported as being used throughout a therapy session by the highest percentage of providers: responding to a child's attempts at communicating (72.70\%); using motivators for teaching opportunities $(69.70 \%)$; using positive affect and animation (69.10\%); and modeling appropriate communication $(68.70 \%)$. Alternatively, the following NDBI strategies were reported as not being used within a therapy session by the highest percentage of providers: guiding the pace and frequency of direct teaching (19.70\%); setting up the activity space for success/limiting distractions $(9.90 \%)$; using contingent and natural social reinforcements $(7.60 \%)$; and supporting a correct response when prompting $(6.10 \%)$. Although, overall, it appeared that providers reported embedding naturalistic and developmental strategies more often within their sessions than behavioral strategies, the average use of developmental strategies was positively correlated with the average use of behavioral strategies $(r=0.67, p=0.002)$. Providers' reported use of behavioral strategies was
Table 2 Perceived competency in early intervention approaches for ASD

\begin{tabular}{lll}
\hline & $M(S D)$ & Range \\
\hline Discrete trial training & $2.05(1.26)$ & $1-5$ \\
Early achievements $^{\mathrm{a}}$ & $1.52(0.99)$ & $1-4$ \\
Early start Denver model (ESDM) $^{\mathrm{a}}$ & $2.25(1.25)$ & $1-5$ \\
Enhanced Milieu teaching (EMT) $^{\mathrm{a}}$ & $1.56(0.90)$ & $1-4$ \\
Floortime/DIR model $^{\text {Hanen more than words }}{ }^{\mathrm{a}}$ & $2.43(1.24)$ & $1-5$ \\
Incidental teaching $^{\mathrm{a}}$ & $1.99(1.17)$ & $1-5$ \\
JASPER $^{\mathrm{a}}$ & $2.49(1.48)$ & $1-5$ \\
Preschool autism communication therapy $^{\text {PACT) }}$ & $3.54(1.32)$ & $1-5$ \\
Pivotal response training (PRT) $^{\mathrm{a}}$ & $1.33(0.76)$ & $1-4$ \\
PLAY Project $^{\mathrm{a}}$ & & \\
Project ImPACT $^{\mathrm{a}}$ & $1.66(1.15)$ & $1-5$ \\
Reciprocal imitation training $^{\mathrm{a}}$ & $1.47(0.86)$ & $1-4$ \\
SCERTS/early social interaction project $^{\mathrm{a}}$ & $1.23(0.58)$ & $1-4$ \\
Social ABCs & $1.86(1.30)$ & $1-5$ \\
TEACCH $_{\text {Verbal behavior }}^{\mathrm{a}}$ & $1.52(0.89)$ & $1-4$ \\
\hline
\end{tabular}

${ }^{\mathrm{a} D e n o t e s ~ N D B I ~ p r o g r a m ~}$

positively correlated with their reported competency in an NDBI program $(r=0.31 ; p=0.01)$. Providers' reported use of developmental strategies was not correlated with perceived NDBI competence $(r=0.15 ; p=0.23)$ (Fig. 1).

EI providers' qualitative description of how they support young children with or having an increased chance of having ASD fell into four overarching themes: (1) intervention goals being parent-led; (2) having little formal training in manualized NDBI programs; (3) using a blend of broader NDBI strategies; and (4) perceiving the need to adapt manualized NDBI programs to meet the needs of the EI system. All primary themes and their definitions are displayed in Table 3.

\section{Intervention Goals Being Parent-Led}

When describing how they support families of at-risk children, many EI providers shared that the goals that they targeted and the strategies that they used were strongly influenced by caregiver preferences. They indicated that this is a core feature of the EI delivery system but also, at times, could be a barrier to delivering NDBI programs and/or strategies that might not align with caregiver preferences.

Even if I am the person writing the IFSP, early intervention is so driven by where the parents want to go that I can lean and guide and suggest and recommend, but I don't always get to build exactly what I want. 
Fig. 1 Provider reported NDBI strategy use within a recent session with a specified at-risk child

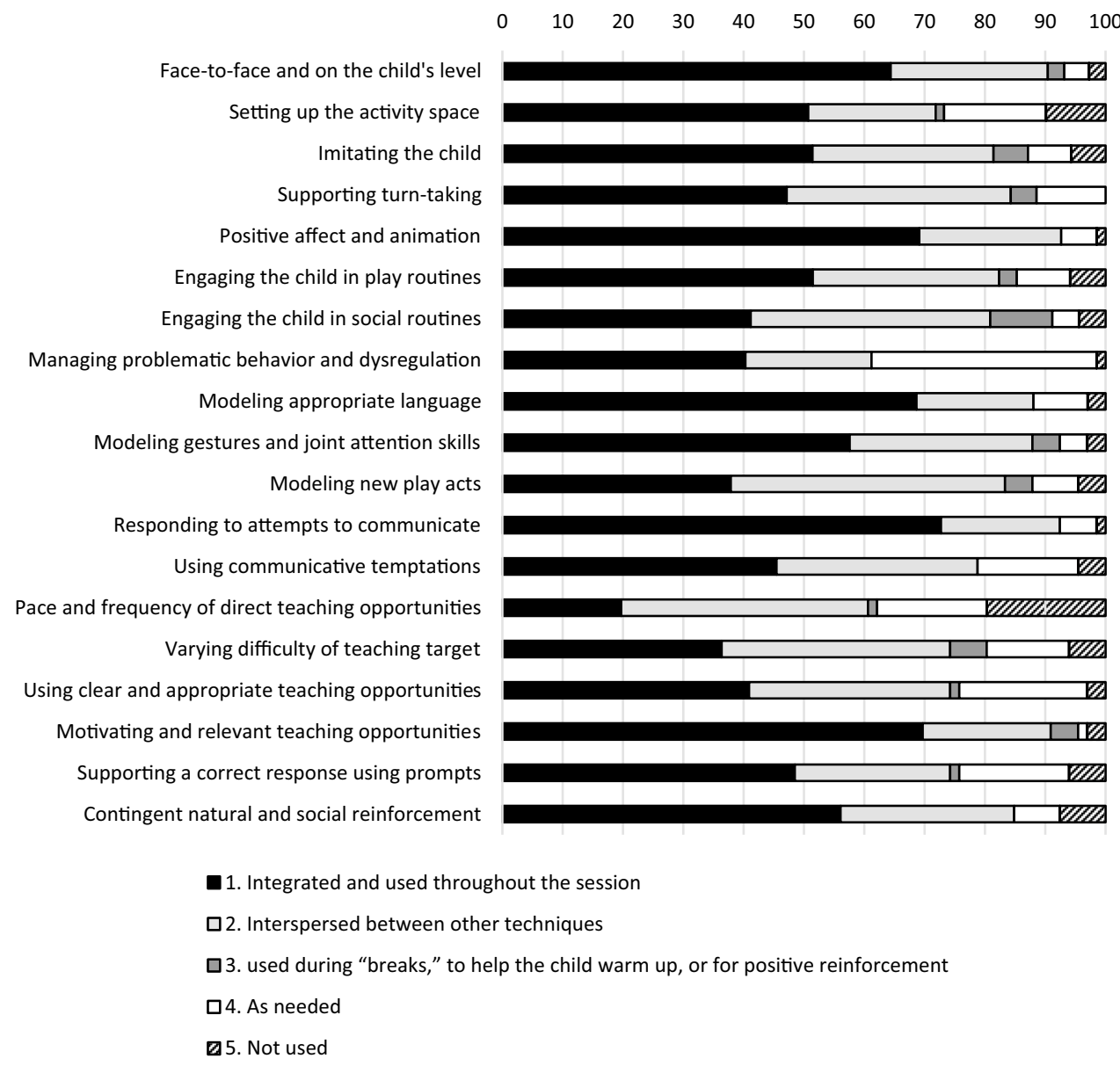

\section{Having Little Formal Training in Manualized NDBI Programs}

Although EI providers within one county reported receiving training in a specific manualized NDBI program (i.e., ESDM), the vast majority of providers shared that they had no formal training in delivering these programs.

I would say that my access to specific programs is really limited, right? And so, other than what I learned, I mean I took an RBT course [non-NDBI] just because I wanted to see and I feel like I've picked up snippets along the way but I would never say that I am trained in the Early Start model or I'm fully certified in Floortime [nonNDBI].

I think I would say that in this area of the state there is very little formal autism training in any capacity. I think some of us who have been in the field for a while have come to learn how to support these kids on our own initiatives.

\section{Using a Blend of Broader NDBI Strategies}

Instead of delivering manualized NDBI programs, EI providers reported using a variety of strategies to support the social communication development of toddlers that they perceived to have an increased likelihood of having ASD. Providers indicated not necessarily having a name for these strategies per se, but feeling as though they are equipped to deliver them both directly to a child and in a parent-mediated intervention format.

And then in terms of strategies, okay, it's funny because as I was doing the survey, I was like, 'I don't know what the names of any of these program things are, but I do this strategy, I do that, I do this.' Do I do it exactly right? I have no idea but I'm flying off what I can, you know?

Something similar, child-led, play-based, teach through play, but I will use different modalities, um, 
Table 3 Primary themes, definitions, and examples by study aim

\begin{tabular}{|c|c|}
\hline Theme & Definition \\
\hline \multicolumn{2}{|l|}{ Aim 1} \\
\hline Intervention goals being caregiver-led & $\begin{array}{l}\text { Goals of the services that are provided are guided by caregivers, thus, at times making } \\
\text { it difficult to deliver NDBI programs and strategies }\end{array}$ \\
\hline No formal NDBI training & Providers reporting not having formal training in an NDBI program \\
\hline Using broader NDBI strategies & $\begin{array}{l}\text { Providers described the broad use of NDBI strategies but not a name or cohesive } \\
\text { framework for these strategies }\end{array}$ \\
\hline Needing to adapt NDBI programs & $\begin{array}{l}\text { Providers described the need to adapt NDBI programs to fit the EI system and child } \\
\text { needs. They described a variety of ways that they might adapt formal programs in } \\
\text { response to individual child needs }\end{array}$ \\
\hline \multicolumn{2}{|l|}{ Aim 2} \\
\hline NDBI program knowledge & $\begin{array}{l}\text { Provider knowledge of NDBI programs impacting their ability to seek additional train- } \\
\text { ing }\end{array}$ \\
\hline County service delivery model & $\begin{array}{l}\text { Whether a county uses independent contractors versus a specialty ASD team impacts } \\
\text { the funding and incentive for providers to attend training }\end{array}$ \\
\hline State- and county-level guidelines & $\begin{array}{l}\text { Overarching guidelines are needed to support providers in knowing what interventions } \\
\text { are best used for children with an increased likelihood of having ASD }\end{array}$ \\
\hline $\begin{array}{l}\text { Barriers tied to using manualized ASD programs } \\
\text { when working with children who may not have an } \\
\text { ASD diagnosis }\end{array}$ & $\begin{array}{l}\text { The dilemma of delivering NDBI programs and strategies that have ASD terminology } \\
\text { to families who are perceived as not being ready to hear that their child might be } \\
\text { at-risk }\end{array}$ \\
\hline \multicolumn{2}{|l|}{ Aim 3} \\
\hline More training and knowledge & $\begin{array}{l}\text { Providers describing the need for more training and continuing education in evidence- } \\
\text { based early intervention for young children with an increased chance of having ASD }\end{array}$ \\
\hline State- or county-level resources necessary for training & $\begin{array}{l}\text { The need for funding, time, and additional incentives that would permit providers to } \\
\text { attend training efforts }\end{array}$ \\
\hline $\begin{array}{l}\text { The benefits and challenges of being trained in a } \\
\text { manualized NDBI programs versus in broader NDBI } \\
\text { strategies }\end{array}$ & $\begin{array}{l}\text { Providers' opinions regarding whether training efforts should be specific to a manual- } \\
\text { ized NDBI program or broader strategies }\end{array}$ \\
\hline
\end{tabular}

I'm working on my BCBA, so I've got that slant, um, so yeah it um varies.

\section{Perceiving the Need to Adapt Manualized NDBI Programs to Meet the Needs of the El System}

Finally, EI providers consistently reported feeling the need to adapt manualized NDBI programs when working with families of young children with social communication delays. This was true for providers both formally trained and untrained in using these manualized curriculums. Providers highlighted a variety of types of adaptations that fit within this overarching theme. A few examples included a need to tailor program content in response to the needs of individual families/children, and the need to blend strategies or materials across different manualized NDBI programs.

Think of if I knew all of it [NDBI programs], granted I might never fully subscribe to one program, but to be able to know all the nooks and crannies and pieces and to pull from it and do the pieces that work for families, it would be really nice, right? (Example of overarching theme of program adaptation).
Not every program fits every child so I think that's the benefit of learning different programs because then you can kind of tailor it to the child. (Example of "tailoring program content").

If a parent is having a hard time understanding that social communication piece with the child, like I took a Hanen course and there was a really nice handout talking about the level of communication so I will pull that in because it's helpful to the parent, you know, its meaningful or I'll slip in a strategy from something else because it matches what we are doing that week. (Example of "blending program strategies").

\section{Factors Impacting NDBI Program and Strategy Use}

Qualitatively, providers described that the following factors impacted their use of manualized NDBI programs and/or broader NDBI strategies: (1) NDBI program knowledge; (2) the county service delivery model; (3) state- and countylevel guidelines; and (4) barriers tied to using manualized ASD programs for children who may not yet have an ASD diagnosis. 


\section{NDBI Program Knowledge}

A large number of providers shared that a primary barrier to seeking out additional training in manualized NDBI programs, and other early intervention models, was their own knowledge of these programs. Although providers indicated being familiar with some manualized interventions, others shared that the manualized NDBI programs listed in the survey were unfamiliar to them.

For me, when I was reading the survey, I was like, 'let me google this,' and most of them were new but it was neat to see that most of them had training models for them.

\section{The Delivery Model of County El Services}

Providers consistently emphasized that their county's service delivery model played a significant role in ongoing training and continuing education opportunities, including those specific to NDBI programs and strategies. Providers within many counties indicated that their county used an independent contractor model to deliver EI services. By being independent contractors rather than employees, participants reported that they are paid hourly and, as such, attending any formal training is both a significant cost and direct loss of income.

So, the director of our CCB, she sends emails sometimes, 'Hey there is this training going on somewhere or online,' but, again, contractors only get paid when working with a child so that's just not going to happen. So, say someone who is really interested in these strategies that have been proven to be effective, they would need to find a way to get that training, pay for it themselves, and then also miss out on time with the kids on their caseload. You know, so there are all of these reallife implications that make it really difficult for people to continue doing that, which is another facet of being independent contractors versus employees. You know, because if you were an employee, you could go to a training and you would still be getting paid whereas that is not the case being an independent contractor.

This was contrasted with one specific county that reported having their own ASD team with funding and incentives to attend ongoing training opportunities. They shared that this additional funding and time permitted the county to bring in training for manualized NDBI programs, and to support providers in attending these training.

[County name] brings in as many opportunities as they can in house so that we can do the group trainings, um, the ESDM had-she brought in a one-day review for those that hadn't gotten the class and ... a lot of us could sign up and usually it's for a nominal fee or for free, um, but we have a lot of opportunities, they really support us in that continuing education piece.

\section{State- and County-Level Support}

In addition to barriers associated with being independent contractors, many providers emphasized that there are not overarching guidelines at both the county and state-level regarding how best to serve young children at-risk for ASD. They shared that this can make it difficult to know how best to support young children with social communication delays and what intervention models would be important to be trained in.

So, I think that's one hard part of it, unless there is some leadership and guidance for those programs then taking the time and energy and money, all those resources to go and get certified on something when you may or may not be able to use because it's not supported is a huge barrier.

\section{Barriers Tied to Using Manualized ASD Programs When Working With Children Who May Not Have an ASD Diagnosis}

Finally, providers indicated that it can be difficult to use both formal NDBI programs and broader NDBI strategies when working with families within the EI system who appear as though they are not ready to hear that their child has an increased chance of having ASD. Due to this issue, participants voiced concern that manualized programs that use ASD terminology might result in difficulty engaging parents.

With some families, you'll lose them and then they'll just close the door. That's the last thing that we want to see happen and I think that is something that comes with experience, we have had families where the relationship is not there, and it comes too soon and they are gone.

You know and there is this really difficult line to walk around how am I going to be kind and honest and informed without scaring people? How am I going to get across in an hour and a half that I really want your kid to talk but there is so much that needs to happen before that can happen?

\section{Methods to Increase the Delivery of NDBI Programs and Strategies}

The majority of providers who participated in focus groups and individual interviews reported the need for: (1) more training and knowledge in how to provide intervention to young children with or at-risk for ASD. When asked about 
the most appropriate way to receive this training, providers talked extensively about: (2) state- or county-level resources necessary for training; and (3) the benefits and challenges of being trained in a manualized NDBI program versus in broader NDBI strategies.

\section{More Training and Knowledge}

By and large, EI providers reported that there was great need for better training opportunities regarding evidence-based intervention for young children with social communication delays. This was true for providers both trained and untrained in manualized NDBI programs.

A lot of them (programs), to be honest, I didn't know what you were talking about because of the acronyms and stuff. Like I might know the strategy, but I didn't know the acronyms. I just left that survey feeling really hungry to learn more.

But we know that more education is better, and I would love to get training. I would love to feel like I had even more tools in my toolbox, so, I can't speak for all early interventionists but I would say that the majority of people that I collaborate with or work alongside in any way, shape, or form is probably in the same boat.

\section{State- or County-Level Resources that Would Be Necessary for Training}

Despite consistently reporting the need for training opportunities, providers also emphasized the resources that would be necessary at the county- and state-level for this training to occur. The resources most frequently cited included funding to attend training, built in time-off to attend training, and the ability to receive follow-up consultation after attending a training.

I'll tell you what I would like to see. I feel like there should be some grants or incentives to support learning a bit more, um, and then being able to be focused and get applied practice in it, um, in whatever way that looks in terms of time and program.

\section{The Benefits and Challenges of Being Trained in a Manualized NDBI Programs Versus in Broader NDBI Strategies}

When asked about their opinion on being trained in a manualized NDBI program versus broader NDBI strategies, providers generally talked about the benefits of being trained in manualized programs. When discussing these benefits, some providers highlighted that these programs may be better at supporting caregiver learning due to providing a more coherent framework for what caregivers are learning and why.
But, yeah, the benefit of those programs is that there is more of a guideline and something to follow to keep you on track as a provider and to make sure parents are learning in a way that makes sense.

My biggest pet peeve is that we give parents these big picture suggestions without actually giving them examples and showing them what they mean. And by having a curriculum, there is better guidance on exactly what the strategies are, how parents can implement them, and how they all fit together.

Providers also clarified who might benefit most from being trained in manualized programs and shared that providers may benefit most from these curricula when they have less experience working with children at-risk for ASD. These providers suggested that, as EI providers gained experience, it would then be easier to adapt the manualized programs that they had previously learned in order to meet the needs of different children and families.

I think it depends how long you've been in the field, you know, so if you have someone who's new and hasn't been trained, sometimes having a curriculum is best for fidelity purposes because then you're more consistent and you can follow through and something builds on something.

Finally, a couple of providers described that manualized programs may be more effective than using blended NDBI strategies, given the larger evidence-base to support these curricula.

I know there was some research done in terms of preschool that kids aren't necessarily as effective in their growth patterns when you are piece-mealing it because you don't have the consistency.

\section{Discussion}

Results from this study provide insight into how providers naturally support young children with ASD in addition to those with an increased likelihood of having ASD within an EI system. Qualitative results helped to expand upon quantitative findings from an NDBI strategy survey. That is, quantitatively, providers reported relatively high confidence in supporting children with an increased chance of having ASD through the delivery of broader NDBI strategies. Within the NDBI strategy survey, EI providers appeared somewhat more likely to report using developmental strategies when working with a child with social communication delays. Interestingly, providers who reported greater use of behavioral strategies also tended to report greater competence in using manualized NDBI programs. This may suggest the utility of these programs in supporting behavioral strategy 
use. However, providers' overall report of developmental and behavioral strategy use was correlated, suggesting the use of a range of NDBI strategies when supporting at-risk children.

Although quantitative findings underscored the existing use of NDBI strategies within the EI system, qualitative findings highlighted variability in how providers deliver these strategies to families. Specifically, the vast majority of providers described using broader NDBI strategies in a manner that was not encompassed by a manualized framework. Additionally, although EI providers clearly stated a need for training in manualized NDBI programs and broader NDBI strategies, qualitative results were critical in highlighting factors that might impact the training in and implementation of these models. Specifically, EI providers almost unanimously described that their training in NDBI programs and/ or strategies would be impacted by system-level factors, such as their county's service delivery model. With the exception of one county with a specialty ASD team, many counties were reported to contract with independent EI providers who were paid hourly. Without a formal salary or funding to attend training, EI providers who were independent contractors emphasized that it was not feasible or cost-effective to be trained in manualized NDBI programs.

Finally, qualitative methods permitted asking EI providers explicitly about their preferences tied to receiving training in a manualized program versus broader NDBI strategies. In response to these questions, participants more often talked about the benefits of learning manualized programs given the frameworks that they use to support provider fidelity and parent learning. However, many providers referenced that training in a manualized program might be most applicable when new to supporting children with ASD and that, ultimately, this training would permit providers to adapt these programs as they became more familiar with them. A number of providers voiced that this tailoring would be essential given the family-centered emphasis of EI services. They also shared that many manualized NDBI programs explicitly reference ASD and/or the social communication delays associated with ASD, and that this terminology might be negatively received by families, leading them to discontinue EI services.

\section{Implications}

This study provides insight into usual care for young children with an increased chance of having ASD who are served within the EI system. This insight is important given the large majority of young children who are served within this system prior to receiving a medical diagnosis of ASD (Monteiro et al. 2016). Overall, it is promising that providers reported using NDBI strategies, thus, highlighting the inherent strength and capacity of providers within this system. Interestingly, within the context of the survey, providers shared most often embedding naturalistic and developmental strategies throughout their sessions. The higher reported use of naturalistic and developmental strategies may more closely align with EI provider pre-service training. However, given the strong evidencebase for the use of behavioral strategies, this finding may reflect greater need for support in using strategies aimed at directly teaching new skills.

In addition to providers describing the need for more training in NDBI programs and strategies, this study also underscores the importance of proactively considering factors that may impact EI providers' ability to both attend in-service trainings and to implement NDBI programs and strategies following training efforts. The factors discussed by EI providers within this study align relatively well with a variety of implementation frameworks in that providers described the importance of considering factors specific to the NDBI programs themselves, factors specific to EI providers (e.g., self-efficacy around program knowledge), and system-level factors (e.g., funding, service delivery model, program guidelines; Damschroder et al. 2009; Glasgow et al. 2019). In fact, it was the system-level factors that providers most often emphasized as needing to be addressed in order to move forward with more comprehensive training. Prior to in-service training efforts, it would be imperative to partner with state- and/or countylevel administrators and providers to set in place guidelines, incentives, and resources to permit training efforts. Having these structures in place may maximize the reach, impact, and sustainment of in-service training. Although not assessed within this study, it will also be important to understand the impact of more explicit pre-service training.

As stated, EI providers discussed the inherent benefits of being trained in a manualized NDBI curriculums while also consistently reporting the need to adapt these programs in response to individual child needs and family goals. This finding is not necessarily surprising given the known tendency of community providers to adapt evidence-based practices when delivered within both controlled trials (Rogers et al. 2020b) and community contexts (Stirman et al. 2013). In fact, these adaptations are thought to be at the foundation of program sustainment (Chambers et al. 2013; Shelton et al. 2018). However, the juxtaposition between the clear desire to learn a manualized program yet the emphasis on adapting them was striking. Although providers described some seemingly minor adaptations (e.g., slight changes to terminology; repeating core content), it was unclear how other adaptations might impact program effectiveness (e.g., blending manualized NDBI programs). 


\section{Future Directions}

Although the results of this study highlight how intervention is reportedly provided to young children with and at-risk for ASD within the EI system, there is great need to better understand NDBI strategy use in a more objective and holistic manner. For example, within this study, providers quantified their use of all NDBI strategies and, in doing so, generally reported greater use of developmental strategies than behavioral strategies. However, rather than quantifying strategy use, it will be imperative to understand how providers use NDBI strategies together, as NDBI programs use a blend of strategies that is dependent on the specific program model, a child's engagement and behavior, as well as the intervention setting. Therefore, more frequent use of any given strategy may not necessarily equate to better quality or outcomes. The use of observational fidelity measures may be useful to capture a more holistic view of NDBI strategy use within the EI system (Frost et al. 2020).

In addition to understanding the relative use of NDBI strategies, it will also be important to dive deeper into the adaptations that community providers make to existing manualized NDBI programs, the rationale for these adaptations and, importantly, the impact that these adaptations have on parent and child outcomes (Georgiadis et al. 2020; Kirk et al. 2020). Understanding adaptation within the EI system is particularly important given the system's emphasis on providing services that are responsive to family needs and goals. Therefore, in addition to understanding cliniciandriven adaptations, it may be important to understand the role of shared adaptation between families and providers within the EI system. Examining the impact of these adaptations more thoroughly may build our understanding of how these programs can be flexibly delivered in a way that both retains their impact while also permitting their scale-up and sustainment within a family-centered system (Georgiadis et al. 2020).

Finally, providers clearly reported the dilemma around delivering a manualized NDBI to children who may not yet have an ASD diagnosis. The discrepancy between the potential of NDBI programs and strategies to meet a broader need (Maye et al. 2020) and the relatively narrow scope and terminology that is embodied within programs may need to be addressed if they are to be used on a larger scale.

\section{Limitations}

There are important limitations to consider within this study. The first is that the results of this study reflect the perceptions of EI providers within one state. Given the variability in EI models across states, the results of this study may not generalize. This study also exclusively examined provider-reported delivery of manualized NDBI programs and strategies. Although there is research to suggest that community providers' report of intervention strategies is positively correlated with their actual use of strategies (BrookmanFrazee et al. 2020), it is possible that the study data are not wholly reflective of how providers actually support young children with or at-risk for ASD.

Additionally, given the manner in which providers reported on their strategy use, it was not possible to examine how providers used NDBI strategies together. This is an important limitation given that greater overall strategy use does not necessarily indicate that an intervention is being delivered at a higher fidelity and/or quality. As previously stated, future research is needed to more thoroughly understand the relative use of these strategies within the EI system, and for which children certain strategies are more likely to be used. Finally, this study did not examine parent perspectives regarding the receipt of NDBI programs and strategies, and did not examine training that EI providers may have received pre-service.

\section{Conclusion}

There has been growing interest in more efficiently translating evidence-based, NDBI programs within the EI system given the proportion of young children with an increased likelihood of having ASD being served within this system. There is also growing recognition that NDBI programs and/ or strategies may be able to serve a much broader group of young children, not exclusively those with ASD or social communication delays (Maye et al. 2020). Despite this interest, there is also great need to understand EI providers' existing use of both specific NDBI programs, as well as the broader strategies that make up these programs. This study highlights providers' reported use of manualized NDBI programs and strategies within the EI system, as well as factors that impact their ability to do so. This knowledge provides important insight into the inherent strengths and limitations of EI providers and systems, and foundational next steps to support the translation of early interventions that are both evidence-based and appropriately flexible to meet the needs of the EI system.

Acknowledgments We would like to acknowledge the tremendous support of Early Intervention Colorado throughout this research project. This includes support from Beth Cole, Ed.D., and the providers, service coordinators, and administrators who dedicated time and resources to organize, participate, and disseminate the results of this research. We would also like to thank JFK Partners, the LEND and UCEDD in Colorado, for dissemination support.

Author Contributions KP contributed to conceptualization, methodology, data colleciton, data analysis, writing-original draft, writingreview \& editing, project administration. HM contributed to data 
collection and analysis, writing-original draft, writing-review \& editing. KF contributed to data collection, analysis, writing-review \& editing. JR contributed to conceptualization, writing-review \& editing, supervision. BI contributed to conceptualization, writing-review \& editing, supervision.

Funding This manuscript has not been previously published in any form, does not contain copyrighted material, and is not under review elsewhere. The authors have no funding to disclose.

\section{Compliance with Ethical Standards}

Conflict of Interest The authors have no potential conflicts of interest to disclose.

Ethical Approval The research presented in this manuscript adhered to basic ethical considerations for the protection of human participants in research.

Informed Consent Informed consent was obtained from all participants.

\section{References}

Adams, R. C., \& Tapia, C. (2013). Early intervention, IDEA Part C services, and the medical home: Collaboration for best practice and best outcomes. Pediatrics, 132, e1073-e1088. https://doi. org/10.1542/peds.2013-2305.

Angell, A. M., Empey, A., \& Zuckerman, K. E. (2018). A review of diagnosis and service disparities among children with autism from racial and ethnic minority groups in the United States. International Review of Research in Developmental Disabilities, 55, 145-180. https://doi.org/10.1016/bs.irrdd.2018.08.003.

Booth, A., Hannes, K., Harden, A., Noyes, J., Harris, J., \& Tong, A. (2014). COREQ (consolidated criteria for reporting qualitative studies). Guidelines for Reporting Health Research: A User's Manual. https://doi.org/10.1002/9781118715598.

Bottema-Beutel, K., Kapp, S. K., Lester, J. N., Sasson, N. J., \& Hand, B. N. (2020). Avoiding ableist language: Suggestions for autism researchers. Autism in Adulthood. https://doi.org/10.1089/ aut.2020.0014.

Brookman-Frazee, L., Stadnick, N. A., Lind, T., Roesch, S., Terrones, L., Barnett, M. L., et al. (2020). Therapist-observer concordance in ratings of EBP strategy delivery: Challenges and targeted directions in pursuing pragmatic measurement in children's mental health services. Administration and Policy in Mental Health and Mental Health Services Research. https://doi.org/10.1007/s1048 8-020-01054-x.

Chambers, D. A., Glasgow, R. E., \& Stange, K. C. (2013). The dynamic sustainability framework: Addressing the paradox of sustainment amid ongoing change. Implementation Science, 8(1), 117. https:// doi.org/10.1186/1748-5908-8-117.

Creswell, J. W. (2013). Qualitative inquiry and research design: Choosing among five approaches. Thousand Oaks, CA: Sage.

Damschroder, L. J., Aron, D. C., Keith, R. E., Kirsh, S. R., Alexander, J. A., \& Lowery, J. C. (2009). Fostering implementation of health services research findings into practice: A consolidated framework for advancing implementation science. Implementation Science, 4(1), 1-15. https://doi.org/10.1186/1748-5908-4-50.

Dawson, G. (2008). Early behavioral intervention, brain plasticity, and the prevention of autism spectrum disorder. Development and
Psychopathology, 20, 775-803. https://doi.org/10.1017/S0954 579408000370.

Eisenhower, A., Pedraza, F. M., Sheldrick, R. C., Frenette, E., Hoch, N., Brunt, S., \& Carter, A. S. (2020). Multi-stage screening in early intervention: A critical strategy for improving ASD identification and addressing disparities. Journal of Autism and Developmental Disorders. https://doi.org/10.1007/s10803-020-04429-z.

Fox, S. E., Levitt, P., \& Nelson, C. A., III. (2010). How the timing and quality of early experiences influence the development of brain architecture. Child Development, 81(1), 28-40. https:// doi.org/10.1111/j.1467-8624.2009.01380.x.

Frost, K. M., Brian, J., Gengoux, G. W., Hardan, A., Rieth, S. R., Stahmer, A., \& Ingersoll, B. (2020). Identifying and measuring the common elements of naturalistic developmental behavioral interventions for autism spectrum disorder: Development of the NDBI-Fi. Autism. https://doi.org/10.1177/1362361320944011.

Frost, K. M., \& Ingersoll, B. (2020). Utilization of developmental and behavioral intervention techniques in usual care: Initial validation of a self-report rating scale in a sample of applied behavior analysis providers. Montreal: International Meeting for Autism Research.

Georgiadis, C., Peris, T. S., \& Comer, J. S. (2020). Implementing strategic flexibility in the delivery of youth mental health care: A tailoring framework for thoughtful clinical practice. Evidence-Based Practice in Child and Adolescent Mental Health, 5(3), 215-232. https://doi.org/10.1080/23794925.2020.17965 50 .

Glasgow, R. E., Harden, S. M., Gaglio, B., Rabin, B., Smith, M. L., Porter, G. C., et al. (2019). RE-AIM planning and evaluation framework: Adapting to new science and practice with a 20-year review. Frontiers in Public Health, 7(64), 1-9. https:// doi.org/10.3389/fpubh.2019.00064.

Guest, G., Bunce, A., \& Johnson, L. (2006). How many interviews are enough?: An experiment with data saturation and variability. Field Methods, 18, 59-82. https://doi.org/10.1177/1525822X05279903.

Hsieh, H. F., \& Shannon, S. E. (2005). Three approaches to qualitative content analysis. Qualitative Health Research, 15(9), 1277-1288. https://doi.org/10.1177/1049732305276687.

Kaiser, A. P., \& Hancock, T. B. (2003). Teaching parents new skills to support their young children's development. Infants and Young Children, 16(1), 9-21.

Kendall, P. C., \& Beidas, R. S. (2007). Smoothing the trail for dissemination of evidence-based practices for youth: Flexibility within fidelity. Professional Psychology: Research and Practice, 38(1), 13. https://doi.org/10.1037/0735-7028.38.1.13.

Kirk, M. A., Moore, J. E., Stirman, S. W., \& Birken, S. A. (2020). Towards a comprehensive model for understanding adaptations' impact: The model for adaptation design and impact (MADI). Implementation Science, 15, 1-15. https://doi.org/10.1186/s1301 2-02001021y.

Lau, A., Barnett, M., Stadnick, N., Saifan, D., Regan, J., Wiltsey Stirman, S., et al. (2017). Therapist report of adaptations to delivery of evidence-based practices within a system-driven reform of publicly funded children's mental health services. Journal of Consulting and Clinical Psychology, 85(7), 664. https://doi.org/10.1037/ ccp0000215.

Maenner, M. J., Shaw, K. A., \& Baio, J. (2020). Prevalence of autism spectrum disorder among children aged 8 years: Autism and developmental disabilities monitoring network, 11 sites, United States, 2016. MMWR Surveillance Summaries, 69(4), 1-12. https ://doi.org/10.15585/mmwr.ss6904a1.

Maye, M., Sanchez, V. E., Stone-MacDonald, A., \& Carter, A. S. (2020). Early interventionists' appraisals of intervention strategies for toddlers with autism spectrum disorder and their peers in inclusive childcare classrooms. Journal of Autism and 
Developmental Disorders. https://doi.org/10.1007/s10803-02004456-w.pdf.

Miles, M. B., Huberman, A. M., \& Saldana, J. (2014). Qualitative data analysis: A methods sourcebook. Thousand Oaks: Sage.

Monteiro, S. A., Dempsey, J., Broton, S., Berry, L., Goin-Kochel, R. P., \& Voigt, R. G. (2016). Early intervention before autism diagnosis in children referred to a regional autism clinic. Journal of Developmental and Behavioral Pediatrics, 37(1), 15-19. https:// doi.org/10.1097/DBP.0000000000000241.

Nevill, R. E., Lecavalier, L., \& Stratis, E. A. (2018). Meta-analysis of parent-mediated interventions for young children with autism spectrum disorder. Autism, 22(2), 84-98. https://doi. org/10.1177/1362361316677838.

Pierce, K., Gazestani, V. H., Bacon, E., Barnes, C. C., Cha, D., Nalabolu, S., et al. (2019). Evaluation of the diagnostic stability of the early autism spectrum disorderphenotype in the general population starting at 12 months. JAMA Pediatrics, 173(6), 578587. https ://doi.org/10.1001/jamapediatrics.2019.0624.

Rogers, E. M. (2002). Diffusion of preventive innovations. Addictive Behaviors, 27(6), 989-993. https://doi.org/10.1016/S0306 $-4603(02) 00300-3$.

Rogers, S. J., Stahmer, A., Talbott, M., Young, G., Fuller, E., Pellecchia, M., et al. (2020a). Feasibility of delivering parent-implemented NDBI interventions in low resource regions: A pilot randomized controlled study. Journal of Neurodevelopmental Disorders. https://doi.org/10.21203/rs.3.rs-105121/v1.

Rogers, S. J., Yoder, P., Estes, A., Warren, Z., McEachin, J., Munson, J., et al. (2020b). A multisite randomized controlled trial comparing the effects of intervention intensity and intervention style on outcomes for young children with autism. Journal of the American Academy of Child and Adolescent Psychiatry. https:// doi.org/10.1016/j.jaac.2020.06.013.

Sanchez-Garcia, A. B., Galindo-Villardon, P., Nieto-Librero, A. B., Martin-Rodero, H., \& Robins, D. L. (2019). Toddler screening for autism spectrum disorder: A meta-analysis ofdiagnostic accuracy. Journal of Autism and Developmental Disorders, 49, 1837-1852. https://doi.org/10.1007/s10803-018-03865-2.

Schreibman, L., Dawson, G., Stahmer, A. C., Landa, R., Rogers, S. J., McGee, G. G., et al. (2015). Naturalistic developmental behavioral interventions: Empirically validated treatments for autism spectrum disorder. Journal of Autism and Developmental Disorders, 45, 2411-2428. https://doi.org/10.1007/s10803-015-2407-8.

Sheldrick, R. C., Frenette, E., Vera, J. D., Mackie, T. I., MartinezPedraza, F., Hoch, N., et al. (2019). What drives detection and diagnosis of autism spectrum disorder? Looking under the hood of a multi-stage screening process in early intervention. Journal of Autism and Developmental Disorders, 49, 2304-2319. https:// doi.org/10.1007/s10803019-03913-5.

Shelton, R. C., Cooper, B. R., \& Stirman, S. W. (2018). The sustainability of evidence-based interventions and practices in public health and health care. Annual Review of Public Health, 39, $55-76$.

Stahmer, A. C., Brookman-Frazee, L., Rieth, S. R., Stoner, J. T., Feder, J. D., Searcy, K., \& Wang, T. (2017). Parent perceptions of an adapted evidence-based practice for toddlers with autism in a community setting. Autism, 21, 217-230. https://doi. org/10.1177/1362361316637580.

Stewart, R. E., Beidas, R. S., \& Mandell, D. S. (2019). Stop calling them laggards: Strategies for encouraging nonadopters to incorporate evidence-based practices. Psychiatric Services, 70(10), 958-960. https://doi.org/10.1176/appi.ps.201900031.

Stirman, S. W., Miller, C. J., Toder, K., \& Calloway, A. (2013). Development of a framework and coding system for modifications and adaptations of evidence-based interventions. Implementation Science, 8(1), 65. https://doi.org/10.1186/1748-5908-8-65.

Stronach, S. T., \& Schmedding-Bartley, J. L. (2019). Clinical decision making in speech language intervention for toddlers with autism and other social communication delays. Perspectives of the ASHA Special Interest Groups, 4(2), 228-239. https://doi. org/10.1044/2019_PERS-SIG1-2018-0010.

Trembath, D., Gurm, M., Scheerer, N. E., Trevisan, D. A., Paynter, J., Bohadana, G., et al. (2019). Systematic review of factors that may influence the outcomes and generalizability of parent-mediated interventions for young children with autism spectrum disorder. Autism Research, 12(9), 1304-1321. https://doi.org/10.1002/ aur.2168.

Vibert, B. A., Dufek, S., Klein, C. B., Choi, Y. B., Winter, J., Lord, C., \& Kim, S. H. (2020). Quantifying caregiver change across early autism interventions using the measure of NDBI strategy implementation: Caregiver change (MONSI-CC). Journal of Autism and Developmental Disorders. https://doi.org/10.1007/s1080 3-019-04342-0.pdf.

Vivanti, G., Kasari, C., Green, J., Mandell, D., Maye, M., \& Hudry, K. (2018). Implementing and evaluating early intervention for children with autism: Where are the gaps and what should we do? Autism Research, 11(1), 16-23. https://doi.org/10.1002/aur.1900.

Publisher's Note Springer Nature remains neutral with regard to jurisdictional claims in published maps and institutional affiliations. 